\title{
Same Organism, Different Phenotype - Are Phenotypic Criteria Adequate In Coagulase-Negative Staphylococcal Orthopaedic Implant-Associated Infections?
}

\author{
Staffan Tevell1,2® ${ }^{\bowtie}$, Sharmin Baig 3 , Åsa Nilsdotter-Augustinsson ${ }^{4}$, Marc Stegger ${ }^{3}$, Bo Söderquist ${ }^{2}$ \\ 1. Department of Infectious Diseases, Karlstad, and Centre for Clinical Research, County Council of Värmland, Sweden \\ 2. School of Medical Sciences, Faculty of Medicine and Health, Örebro University, Örebro, Sweden \\ 3. Department of Bacteria, Parasites and Fungi, Statens Serum Institut, Copenhagen, Denmark \\ 4. Department of Infectious Diseases and Department of Clinical and Experimental Medicine, Linköping University, Norrköping, Sweden \\ $\triangle$ Corresponding author: Staffan Tevell, Department of Infectious Diseases, Karlstad Hospital, SE-65185 Karlstad, Sweden. Telephone: +4654615000; Fax: \\ +4654616042; staffan.tevell@regionvarmland.se \\ (c) Ivyspring International Publisher. This is an open access article distributed under the terms of the Creative Commons Attribution (CC BY-NC) license \\ (https://creativecommons.org/licenses/by-nc/4.0/). See http://ivyspring.com/terms for full terms and conditions.
}

Received: 2018.09.28; Accepted: 2018.10.28; Published: 2019.01.01

\begin{abstract}
In current diagnostic criteria for implant-associated bone- and joint infections, phenotypically identical low-virulence bacteria in two intraoperative cultures are usually required. Using whole-genome sequencing, we have further characterized three phenotypically different Staphylococcus capitis isolated from one prosthetic joint infection, highlighting the challenges in defining microbiological criteria for low-virulence prosthetic joint infections.
\end{abstract}

\section{Introduction}

Orthopaedic implant-associated bone and joint infections such as prosthetic joint infections (PJIs) and fracture related infections (FRIs) are difficult both to diagnose and to treat. Since there is no gold standard for verifying or excluding infection, several sets of diagnostic criteria exist for PJIs, [1-5] while consensus criteria for the diagnosis of FRIs have only recently been proposed by an international working group. [6] However, microbiological criteria differ between definitions; examples include a) identical (or same) microorganism in two or more intraoperative cultures and/or preoperative aspiration (definitive criteria), b) virulent microorganism (e.g. S. aureus) in one single intraoperative culture or preoperative aspiration (definite or non-existent criteria), and c) any microorganism in one single intraoperative culture (supportive or non-existent criteria).

In a previous study [7], an included patient suffered a chronic knee PJI that presented a sinus tract
1.5 years after the primary arthroplasty, but the prosthesis was not visible, and no other sources of infection were found. Coagulase-negative staphylococci (CoNS) were isolated in all five intraoperative cultures and following the diagnostic procedures at the clinical microbiology laboratory at that time, three of the five samples were further analysed but no determination to species level was performed. No comments about macroscopic colony morphology were noted. The implant was not further analysed, as sonication was unavailable at the laboratory. However, none of these three isolates were phenotypically identical according to antibiotic susceptibility testing, as one isolate (SCPJI10_1-1b) was sensitive to erythromycin, clindamycin, norfloxacin, and ciprofloxacin, the second (SCPJI10_1-1c) was resistant to erythromycin and clindamycin but sensitive to norfloxacin and ciprofloxacin, and the last isolate (SCPJI10_1-1a) was 
resistant to erythromycin, clindamycin, and norfloxacin but sensitive to ciprofloxacin. All other analyses were identical; the isolates were resistant to betalactam antibiotics and gentamicin, and susceptible to fusidic acid, rifampin, trimethoprim/sulfamethoxazole, linezolid and vancomycin (MIC $1.5 \mathrm{mg} / \mathrm{L}$ ), but expressed heterogeneously intermediate susceptibility to glycopeptides according to the Van4-method [7]. The patient was cured using a two-stage revision including antibiotic treatment for three months (vancomycin followed by fluoroquinolone/rifampin combination).

However, the microbiological results pose a clinical challenge; is this a true monomicrobial infection with multiple lineages, or contamination of the intraoperative cultures by different CoNS species? We therefore decided to perform species determination and whole-genome sequencing (WGS) of these isolates, aiming to explore potential similarities and differences of the genomes of three CoNS isolates obtained from different intraoperative tissue cultures from one patient with a PJI.

\section{Methods}

\section{Bacterial isolates}

All three isolates were obtained from tissue biopsies during the same explantation procedure. They were stored at $-70^{\circ} \mathrm{C}$ at the Department of Clinical Microbiology, then subcultured at $35^{\circ} \mathrm{C}$ overnight on Columbia II agar (BD Diagnostic Systems, Sparks, MD, USA) supplemented with $6 \%$ horse blood (SVA, Uppsala, Sweden) and were determined to species level using MALDI-TOF MS (Microflex LT and Biotyper 3.1; Bruker Daltonics, Bremen, Germany).

\section{Whole-genome sequencing (WGS)}

Genomic DNA was purified using a QIAGEN Blood and Tissue Kit, and a sequencing library was produced using Nextera XT (illumina) followed by paired-end sequencing using a 600 cycle kit on a MiSeq sequencer, and have been archived at the European Nucleotide Archive (ENA) with project ID PRJEB29338. Whole-genome sequences were assembled de novo using the SPAdes assembler, and resistance genes were detected with ABRicate using the ResFinder database.

\section{Core genome phylogenetic analyses}

Single-nucleotide polymorphisms (SNPs) were identified using the Northern Arizona SNP pipeline (NASP) with the BWA algorithm to align Illumina reads from individual isolates against the chromosome from S. capitis CR01 (GenBank accession number LN866849) after removal of duplicated regions using NUCmer. Positions with less than 10 -fold coverage and less than $90 \%$ unambiguous variant calls were excluded.

\section{Accessory genome analyses}

Open reading frames were identified and annotated with Prokka v1.12 from the assembled draft genomes. The annotated bacterial genomes were investigated using Roary v3.12 with minimum 85\% similarity on the protein level, and confirmed using reference mapping in the CLCbio Genomics Workbench v11.

\section{Phenotypic analysis}

Biofilm production was analysed using microtitre plate assay (MTP) and Congo red agar (CRA), as well as standard AST by disc diffusion test, as previously described. [7] AST was re-performed for norfloxacin in SCPJI10_1-1a, since the zone diameter was just below the breakpoint, in contrast to the other two isolates. Since the erm $C$ gene was not detected in silico in SCPJI10_1-1a, erythromycin AST was also re-performed for this isolate.

\section{Ethics}

The study was approved by the Regional Ethical Review Board of Uppsala (ref: 2014/418).

\section{Results}

\section{Genomic analysis}

All three isolates were confirmed to be the species $S$. capitis using WGS against the $S$. capitis CR01 chromosome. Analysis revealed no more than three SNPs discriminating these three isolates within the core genome of $91.2 \%(\sim 2.3 \mathrm{mb})$ of the reference, thus considered clonally identical. There were no differences in the accessory genomes, beside the identification of a $2.4 \mathrm{~kb}$ ermC-encoding plasmid identical to pSA7112 (GenBank accession number KX011076) previously characterized in S. aureus.

\section{Biofilm production}

The icaA gene was present in all three isolates, and similar phenotypic biofilm production was demonstrated using MTP and CRA.

\section{Antibiotic susceptibility testing}

Details of the AST are given in Table 1.

\section{Quinolones}

Re-performing the disc diffusion test for norfloxacin demonstrated that the zone diameter for SCPJI10_1-1a remained unchanged, while the zone diameter for SCPJI10_1-1c was found to be 19 millimetres compared to 17 millimetres previously. 
Table 1. Antimicrobial susceptibility testing (AST) using the disc diffusion test: zone size measured in millimetres followed by interpretation according to EUCAST (SIR) for the isolates. Zone diameters and interpretations in parenthesis represent the results from re-performing AST.

\begin{tabular}{|c|c|c|c|c|c|c|c|c|}
\hline Antibiotic & Clindamycin & & Erythromycin & & Norfloxacin & \multicolumn{3}{|c|}{ Ciprofloxacin } \\
\hline EUCAST breakpoint & $22 / 19$ & & $21 / 18$ & & $17 / 17$ & & $20 /$ & \\
\hline SCPJI10_1-1a & 6 & $\mathrm{R}$ & 6 & $\mathrm{R}$ & $16(16)$ & $\mathrm{R}(\mathrm{R})$ & 24 & S \\
\hline SCPJI10_1-1b & 35 & S & 37 & S & 19 & $S$ & 26 & S \\
\hline SCPJI10_1-1c & $6(29)$ & $\mathrm{R}(\mathrm{S})$ & $6(30)$ & $\mathrm{R}(\mathrm{S})$ & $19(17)$ & $S(S)$ & 24 & S \\
\hline
\end{tabular}

However, this did not change the interpretation according to EUCAST breakpoints (accessed 2018-06-26). SNP analysis of the grlA, grlB, gyrA and $g y r B$, and nor $A$ genes, including the promoter region, [8] revealed no differences between the isolates.

\section{Erythromycin/clindamycin}

The erm C gene was not detected in SCPJI10_1-1b or SCPJI10_1-1c, but was found on a plasmid in isolate SCPJI10_1-1a. As SCPJI10_1-1c originally displayed phenotypic resistance while erm $\mathrm{C}$ was not found, this isolate was recovered from the freezer and phenotypic AST and WGS were re-performed. The re-analysis revealed full susceptibility to erythromycin and clindamycin, but the results of re-sequencing were identical to the first analysis and no erm C-containing plasmid was detected.

\section{Discussion}

We present a case of monomicrobial PJI, that initially was identified as polymicrobial since three phenotypically different isolates of $S$. capitis was found, however later confirmed to be identical using WGS. This case highlights the difficulties that may occur in microbiological diagnosis of implant-associated bone and joint infections, and the limitations of present diagnostic definitions. The IDSA definition states that "Two or more intraoperative cultures ... that yield the same organism (indistinguishable based on common laboratory tests including genus and species identification or common antibiogram) may be considered definitive evidence of PJI", but goes on to note that: "The presence of PJI is possible even if the above criteria are not met; the clinician should use his/her clinical judgment to determine if this is the case after reviewing all the available preoperative and intraoperative information". [3] However, data to support the clinician's judgement in this situation are scarce.

Bacteria in a biofilm have complex microbiological characteristics. Diversity by evolution has previously been demonstrated both in morphological phenotype and in antibiotic susceptibility patterns among bacteria in biofilm, [9] including small-colony variants in staphylococci. [10] It is impossible to say whether the norfloxacin resistance detected in one of the isolates in this study is solely due to biological variations around the breakpoints or reflects actual alterations during the growth in biofilm. As for the reversal of erythromycin/clindamycin resistance, loss of plasmid during handling and subculturing seems plausible, as this is a well-described phenomenon. [11] Thus, it seems reasonable to perform AST on all, and not only a selected few CoNS isolates from tissue cultures obtained from infected orthopaedic implants.

In conclusion, differences in phenotype and in antibiotic susceptibility patterns are not reliable enough to disprove a suspected CoNS orthopaedic implant-associated bone and joint infection when multiple cultures show growth of one single, but phenotypically different, species. In addition, different CoNS species seem to be associated with different likelihood of being truly indicative of infection. [12] If one single common set of diagnostic criteria could be developed for each of PJI and FRI, respectively, this may facilitate both clinical care and research in the future. However, more data are needed on the microbiological definition of orthopaedic implant-associated bone and joint infections before reconsidering the microbiological criteria. Until then, communication between clinician and clinical microbiologist is of utmost importance, to ensure access to the best available information when evaluating a patient with suspected CoNS orthopaedic implant-associated infection according to current algorithms.

\section{Acknowledgements}

The authors would like to thank Bengt Hellmark for assistance with sequencing of isolates.

\section{Authors Contributions}

All authors have contributed to the design of the work. ST has collected medical and bacteriological data of the patient. BS performed microbiological analysis of the clinical samples. SB and MS have performed bioinformatic analysis of WGS data. ST wrote the manuscript. SB, ANA, MS and BS made a critical revision of this manuscript. All authors read and approved the final manuscript. 


\section{Funding}

This work was supported by grants from the research committee of Värmland County Council, Sweden (grant numbers LIVFOU-736151 and LIVFOU-776591), and from Nyckelfonden at Örebro University Hospital (OLL-404141).

\section{Competing Interests}

The authors have declared that no competing interest exists.

\section{References}

1. Parvizi J, Zmistowski B, Berbari EF, Bauer TW, Springer BD, Della Valle CJ, et al. New definition for periprosthetic joint infection: from the Workgroup of the Musculoskeletal Infection Society. Clin Orthop Relat Res. 2011; 469: 2992-4.

2. Parvizi J, Gehrke T, Chen AF. Proceedings of the International Consensus on Periprosthetic Joint Infection. Bone Joint J. 2013; 95-B: 1450-2.

3. Osmon DR, Berbari EF, Berendt AR, Lew D, Zimmerli W, Steckelberg JM, et al. Diagnosis and management of prosthetic joint infection: clinical practice guidelines by the Infectious Diseases Society of America. Clinical infectious diseases. 2013; 56: e1-e25.

4. Zimmerli W, Sendi P. Orthopedic Implant-Associated Infections. In Mandell, Douglas, and Bennett's Principles and Practice of Infectious Diseases. Philadelphia, PA: Elsevier/Saunders; 2015: 1328-40.

5. Parvizi J, Tan TL, Goswami K, Higuera C, Della Valle C, Chen AF, et al. The 2018 Definition of Periprosthetic Hip and Knee Infection: An Evidence-Based and Validated Criteria. J Arthroplasty. 2018; 33: 1309-14 e2.

6. Metsemakers WJ, Morgenstern M, McNally MA, Moriarty TF, McFadyen I, Scarborough M, et al. Fracture-related infection: A consensus on definition from an international expert group. Injury. 2018; 49: 505-510.

7. Tevell S, Hellmark B, Nilsdotter-Augustinsson A, Soderquist B. Staphylococcus capitis isolated from prosthetic joint infections. Eur J Clin Microbiol Infect Dis. 2017; 36:115-122.

8. Tanaka M, Wang T, Onodera Y, Uchida Y, Sato K. Mechanism of quinolone resistance in Staphylococcus aureus. J Infect Chemother. 2000; 6: 131-9.

9. Steenackers HP, Parijs I, Dubey A, Foster KR, Vanderleyden J. Experimental evolution in biofilm populations. FEMS Microbiol Rev. 2016; 40: 373-97.

10. Sendi P, Rohrbach $M$, Graber $P$, Frei R, Ochsner PE, Zimmerli W. Staphylococcus aureus small colony variants in prosthetic joint infection. Clinical infectious diseases. 2006; 43: 961-7.

11. Lau BT, Malkus P, Paulsson J. New quantitative methods for measuring plasmid loss rates reveal unexpected stability. Plasmid. 2013; 70: 353-61.

12. Peel TN, Cole NC, Dylla BL, Patel R. Matrix-assisted laser desorption ionization time of flight mass spectrometry and diagnostic testing for prosthetic joint infection in the clinical microbiology laboratory. Diagnostic microbiology and infectious disease. 2015; 81:163-8. 